\title{
Pressurised metered dose inhalers with spacers versus nebulisers for $\beta$-agonist delivery in acute asthma in children in the emergency department
}

\author{
R Boyd, P Stuart
}

Emerg Med J 2005;22:641-642. doi: 10.1136/emj.2003.011296

\begin{abstract}
Objectives: Wet nebulisers are widely used for $\beta$ - 2 agonist delivery in Australasian emergency departments (ED). An increasing body of work suggests that pressurised metered dose inhalers with spacers (pMDI-S) are as effective. This study aims to investigate the effect on admission rates, total time in the ED, and total time in hospital after initiation of routine $\mathrm{pMDI}-\mathrm{S}$ treatment for mild to moderate asthma in children presenting to the ED.

Methods: Children with a discharge diagnosis of asthma were identified over sequential 3 month time periods. In the first period, wet nebuliser treatment was routine and in the subsequent trial period $\mathrm{pMDI}-\mathrm{S}$ treatment was instituted as routine. Admissions rates, total time in hospital, and total time in the ED were recorded for each group.

Results: Admission rates fell significantly from $31 \%$ to $20.6 \%$ after routine use of pMDI-S treatment. There was a nonsignificant trend to an increase in total ED and total hospital times.

Conclusion: Introduction of routine pMDI-S treatment in the paediatric ED results in a significant drop in admission rates but no significant change in total hospital times or total ED times.
\end{abstract}

l nhaled $\beta-2$ agonists are the mainstay of treatment for all asthma severities in both adults and children. Historically, wet nebuliser treatment has been used for children as it has been perceived as easier to administer with superior compliance characteristics This has been especially true for acute treatment. ${ }^{1}$ Conversely, chronic treatment for asthma has relied upon inhaled treatment through pressurised meter dose inhalers using spacers (pMDI-S). Recent work from North America has suggested that, on an individual basis, pMDI-S treatment may be as effective as wet nebuliser treatment in acute mild to moderate asthma. In addition, reevaluation of wet nebuliser treatment has shown significant problems with poor dose delivery. ${ }^{2}$ To date, little work has been published as to the effect of using pMDI-S in Australasian departments as routine treatment for mild to moderate asthma. The aim of this study is to determine the effect on admission rate, total hospital time, and total emergency department (ED) time before and after the initiation of routine use of pMDI-S treatment for mild to moderate asthma in the ED.

\section{METHODS}

This study was undertaken in an urban, mixed ED with an annual census of 43000 attendances of whom 12000 are aged less than 16 years. Data were collected using a departmental patient information system (HAS Edis,
Hospital Administrative Software Solutions Pty, Sydney, Australia). Attendances for children aged 2-14 years with a discharge diagnosis of asthma or wheeze were identified over a three month period. Routine wet nebuliser treatment was the delivery medium for all $\beta$-2 agonists during this period. The dose of $\beta$-agonist used was 2.5-5 mg salbutamol per wet nebuliser treatment. A two week lead in period was then identified during which no data were collected but an extensive departmental education programme was initiated for all medical and nursing staff on delivery methods for mild and moderate asthma. A supply of pMDI-S was introduced to the pharmacy supply and a supply of spacers was made available to the department. The dose administered for each treatment intervention was $600-1200 \mu \mathrm{g}$. This dose was chosen as it has previously been shown to be an optimal therapeutic dose. ${ }^{3}$ A further three month period of data collection was then initiated during which pMDI-S were used as first line treatment in mild to moderate asthma. Departmental guidelines concerning the use of corticosteroids in asthma during the two phases of the study were unchanged-namely, to consider the use of oral prednisolone $1-2 \mathrm{mg} / \mathrm{kg}$ for mild asthma and to mandate the use of oral prednisolone $1-2 \mathrm{mg} / \mathrm{kg}$ for moderate asthma. Data were collected on admission rates to the inpatient paediatric ward, total ED time, and total time in hospital. In addition, reattendances within 48 hours of an initial presentation were monitored. In the analysis, all paediatric presentations with asthma triaged as national triage category 2 (mandating medical attendance within 10 minutes $),{ }^{4}$ and those children admitted to high dependency or intensive care were excluded as they were considered to be outside the mild and moderate categories for asthma. Results were analysed using Pearson $\chi^{2}$ testing and two sample $t$ tests.

\section{RESULTS}

There were 185 presentations in the initial nebuliser treatment period (mean age 6.5 years; 95\% confidence interval (CI) 5.9 to 7.1 , median age 5 years). A total of 165 children presented in the subsequent three month pMDI-S period (mean age 5.7 years; $95 \%$ CI 5.15 to 6.3, median age 4 years) (table 1). The admission rate during the wet nebuliser period was $31 \%$. The admission rate fell to $20.6 \%$ in the subsequent pMDI-S period. This fall was statistically significant on $\chi^{2}$ testing $(\chi=4.92, \mathrm{p}=0.027)$.

With the introduction of pMDI-S, the mean total time spent in the ED rose. A rise from 2 hours 35 minutes (95\% CI 2 hours 21 minutes to 2 hours 49 minutes, median 2 hours 13 minutes) with wet nebulisers to 2 hours 45 minutes (95\% CI 2 hours 18 minutes to 2 hours 47 minutes, median 2 hours and 19 minutes) with the pMDI-S was demonstrated. The

Abbreviations: ED, emergency department; pMDI-S, pressurised metered dose inhalers with spacers 


\begin{tabular}{|c|c|c|c|}
\hline \multicolumn{4}{|l|}{ Table 1} \\
\hline & $\begin{array}{l}\text { Wet nebuliser } \\
\text { treatment }\end{array}$ & $\begin{array}{l}\text { pMDI-S } \\
\text { treatment }\end{array}$ & p Value \\
\hline Admission rate & $31 \%$ & $20.6 \%$ & $p=0.027$ \\
\hline Total time in ED & 155 minutes & 165 minutes & $p>0.05$ \\
\hline $\begin{array}{l}\text { Total time in } \\
\text { hospital }\end{array}$ & 2358 minutes & 3150 minutes & $p>0.05$ \\
\hline $\begin{array}{l}\text { Unscheduled } \\
\text { return rate }\end{array}$ & $1.1 \%$ & $1.2 \%$ & $p=0.12$ \\
\hline $\begin{array}{l}\text { Total number } \\
\text { of children }\end{array}$ & 185 & 165 & \\
\hline
\end{tabular}

rise was not statistically significant. Mean total hospital time for all patients rose from a mean total of 39 hours 18 minutes (95\% CI 30 hours 51 minutes to 47 hours 46 minutes, median 27 hours 39 minutes) in the nebuliser period to 52 hours 30 minutes (95\% CI 40 hours 8 minutes to 64 hours 52 minutes, median 41 hours 40 minutes) with the pMDI-S. Again, this rise was not statistically significant $(\mathrm{p}>0.12)$.

The rate of unscheduled returns to the ED within 48 hours of initial attendance were similar with two children representing within 48 hours in the nebuliser period (2/187, $1.1 \%)$ and two children representing in the pMDI-S period $(2 / 165,1.2 \%)$.

\section{DISCUSSION}

Admission rates for children treated with pMDI-S treatment were lower than those for wet nebuliser treatment. There was no significant rise in total ED although a trend was noted and relatively small numbers may have led to a type II error. This study is similar in its results to North American studies and builds on work suggesting at least equal efficacy for both pMDI-S treatment and wet nebuliser treatment. ${ }^{56}$ This may be because of improved drug delivery as a result of finer particle deposition within the lungs. The pMDI-S may also have benefits in terms of reduced infection control, as it does not produce significant amounts of dispersed respiratory vapour. We have found benefits in both patient and parent training with initiation of pMDI-S treatment in the ED and subsequent discharge with a pMDI-S system for community treatment. The chosen design for this study was a prospective observational method as opposed to a randomised controlled trial. This was chosen so as to study the system wide effects of introducing pMDI-S treatment rather than focus on individual physiological parameters.

This study has a number of limitations. The use of an observational methodology can introduce biases as the before and after groups may not be comparable. In our study, ages alone were matched between the two groups. The training programme instituted to facilitate the changeover to the pMDI-S may have increased the general knowledge levels of medical and nursing staff about the management of acute asthma. However, the training was aimed specifically at pMDI-S use and the departmental guidance on oral corticosteroid treatment and discharge criteria were not altered or made a focus of the departmental teaching programme. Treatment protocols between the ED and the paediatric ward were slightly different throughout both phases of the study. The paediatric ward utilised pMDI-S in all patients over the age of two years who were deemed capable of using the spacer device. This may have introduced bias into the figure for total hospital time as the majority of that time would have been spent in the paediatric ward where pMDI-S was standard treatment. The use of HAS-Edis discharge diagnosis can lead to errors as coding is done by the discharging physician and misdiagnosis can occur. The use of unscheduled returns rates can only be a proxy for patient satisfaction and data were not available concerning attendance at other neighbouring EDs in the 48 hours after attendance at our own department.

\section{CONCLUSION}

Initiating a departmental policy of routine pMDI-S delivery for $\beta-2$ agonists in acute childhood asthma reduces the need for admission and does not significantly increase either total ED times or total hospital times. In addition, there is no change in the rates of unscheduled return to the ED.

\section{Authors' affiliations}

R Boyd, P Stuart, Emergency Department, Lyell McEwin Health Services, Elizabeth Vale SA 5112, South Australia

Competing interests: none declared

Correspondence to: Dr R Boyd, Emergency Department, Lyell McEwin Health Services, Elizabeth Vale SA 5067, South Australia;

Russell.Boyd@nwahs.sa.gov.au

Accepted for publication 23 February 2004

\section{REFERENCES}

1 Blake KV, Hoppe M, Harman E, et al. Relative amounts of albuterol delivered to lung receptors from a metered dose inhaler and nebuliser solution. Chest 1992;101:309-15.

2 Cates CJ, Rowe BH. Holding chamber versus nebulisers for beta-agonist treatment of acute asthma (Cochrane review). In The Cochrane Library, 2000; Issue 2 (CD000052). Oxford Update Software.

3 Dewar AL, Stewart A, Cogswell JJ, et al. A randomised controlled trial to assess the relative benefits of large volume spacers and nebulisers to treat acute asthma in hospital. Arch Dis Child 1999;80:421-23.

4 Australasian College of Emergency Medicine. The Australian Triage Scale. Emerg Med 2002; 14:335-6.

5 Delgado A, Chou K, Silver E, et al. Nebulizers vs metered dose inhalers with spacers for bronchodilator therapy to treat wheezing in children aged 2 to 24 months in a paediatric emergency department. Arch Pediatr Adolesc Med 2003;157(1):76-9.

6 Schuh S, Johnson D, Stephens D, et al. Comparison of albuterol delivered by a metered dose inhaler with spacer versus a nebuliser in children with mild acute asthma. J Pediatr 1999;135(1):22-7. 